\title{
2D MODELING OF ELECTROMAGNETIC WAVES IN COLD PLASMAS
}

\author{
K. Crombé*,†, D. Van Eester*, R. Koch* and V. Kyrytsya* \\ *Laboratory for Plasma Physics, Association "EURATOM - Belgian State" Trilateral Euregio Cluster, \\ Renaissancelaan 30 Avenue de la Renaissance, B-1000 Brussels, Belgium \\ ${ }^{\dagger}$ Department of Applied Physics, Ghent University, Sint-Pietersnieuwstraat 41 B4, B-9000 Ghent, Belgium
}

\begin{abstract}
The consequences of sheath (rectified) electric fields, resulting from the different mobility of electrons and ions as a response to radio frequency (RF) fields, are a concern for RF antenna design as it can cause damage to antenna parts, limiters and other in-vessel components. As a first step to a more complete description, the usual cold plasma dielectric description has been adopted, and the density profile was assumed to be known as input. Ultimately, the relevant equations describing the wave-particle interaction both on the fast and slow timescale will need to be tackled but prior to doing so was felt as a necessity to get a feeling of the wave dynamics involved. Maxwell's equations are solved for a cold plasma in a $2 \mathrm{D}$ antenna box with strongly varying density profiles crossing also lower hybrid and ion-ion hybrid resonance layers. Numerical modelling quickly becomes demanding on computer power, since a fine grid spacing is required to capture the small wavelengths effects of strongly evanescent modes.
\end{abstract}

Keywords: plasmas, wave propagation, sheaths, heating PACS: $52.35 .-\mathrm{g}, 52.50 . \mathrm{Qt}, 52.40 . \mathrm{Kh}, 41.20 . \mathrm{Jb}, 84.40 .-\mathrm{x}$

\section{INTRODUCTION}

Launching radio frequency $(\mathrm{RF})$ power in a plasma with antennas is one of the methods used to achieve the necessary temperatures for nuclear fusion. While very successful in heating the plasma, the method is often accompanied by detrimental effects (material release from walls, local overheating of surfaces and changes in edge transport). The consequences of these so-called sheath (rectified) electric fields, resulting from the different mobility of electrons and ions as a response to radio frequency (RF) fields, are a concern for RF antenna design as it can cause damage to antenna parts, limiters and other in-vessel components. The sheath description in the form of a boundary condition [1] has it limitations; it does not account for a finite plasma density in the sheath layer nor can it describe modifications to the plasma parameters as a result of the sheath fields. In view of a self-consistent description of the dynamics of the plasma and the electromagnetic waves in the presence of metallic objects, a coupling between slow and fast time scale effects will be necessary. Density modifications (on a slow time scale) in front of an RF antenna due to rapidly oscillating electric fields have been explained in [2] by means of a ponderomotive force effect. A 1D description of electromagnetic wave propagation (on the fast time scale) in plasmas with varying densities has been given in [3]. This paper is an extension of [3] to a 2D model.

\section{NUMERICAL EXAMPLES}

A rectangular box of realistic, antenna size, dimensions has been used; the antenna itself is simplified by a current sheet. The cold plasma description has been assumed, as derived in [4]. After eliminating the $\mathrm{E}_{x}$ and $\omega \mathrm{B}_{x}$ components in Maxwell's equations, the wave equations are expressed purely in terms of the components $\mathrm{E}_{y}$ and $\omega \mathrm{B}_{z}$ (which characterize the Transverse Electric, TE, solution to the wave equation w.r.t. the z-direction) and of $\mathrm{E}_{z}$ and $\omega \mathrm{B}_{y}$ (the Transverse Magnetic, TM, solution of the same cold plasma wave equation). The simulation domain is a rectangular box with $(x, y)$-dimensions in the range $\mathrm{H}=0.25-0.60 \mathrm{~m} \times \mathrm{L}=0.50-0.60 \mathrm{~m}$, the antenna frequency has been fixed to $f=51 \mathrm{MHz}$. Simulations are run with a fixed z-component of the wave vector, $k_{z}=5$. The box is filled with a plasma composed of $90 \%$ deuterium and $10 \%$ hydrogen. The antenna is simulated by a constant current sheet in the $y$ direction, intended to preferentially excite the fast wave. Since the cold plasma wave equation is a fourth order system, 4 boundary conditions are needed in each direction to uniquely define a solution. The boundary conditions are 

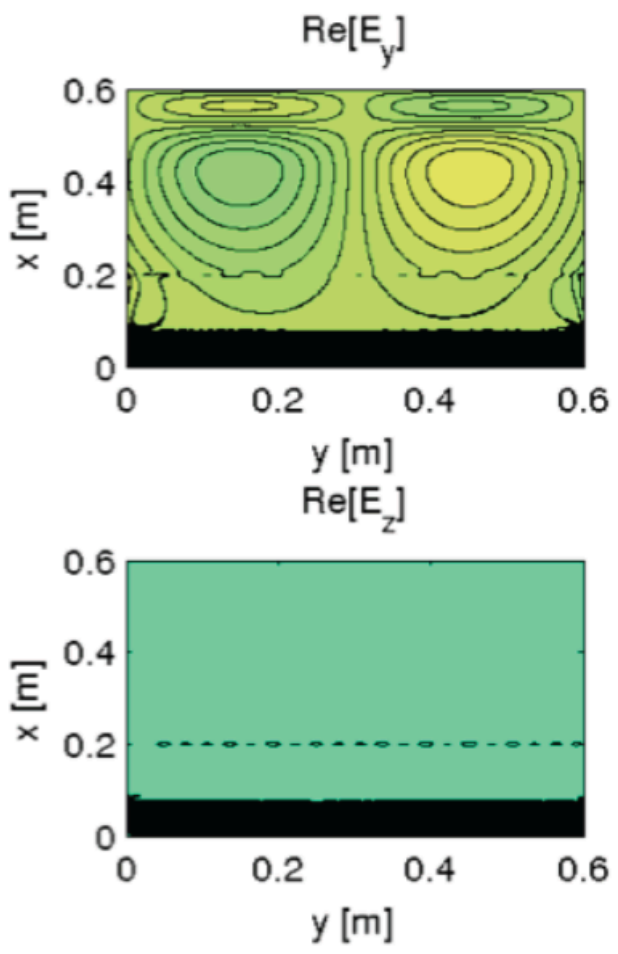
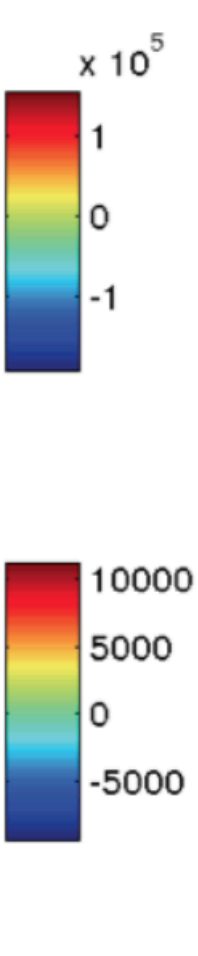

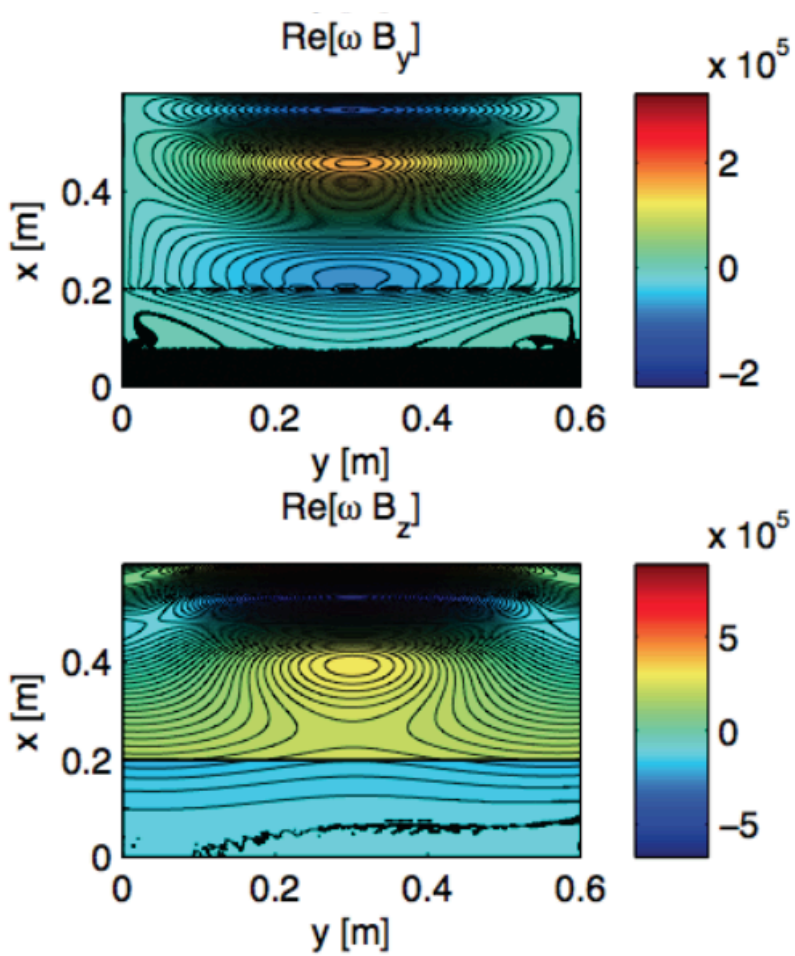

FIGURE 1. Real parts of the electric and magnetic field components in $y$ and $z$ direction for a density variation between $1 \times 10^{15}$ at $\mathrm{x}=0.0 \mathrm{~m}$ to $9 \times 10^{19} \mathrm{~m}^{-3}$ at $\mathrm{x}=0.6 \mathrm{~m}$
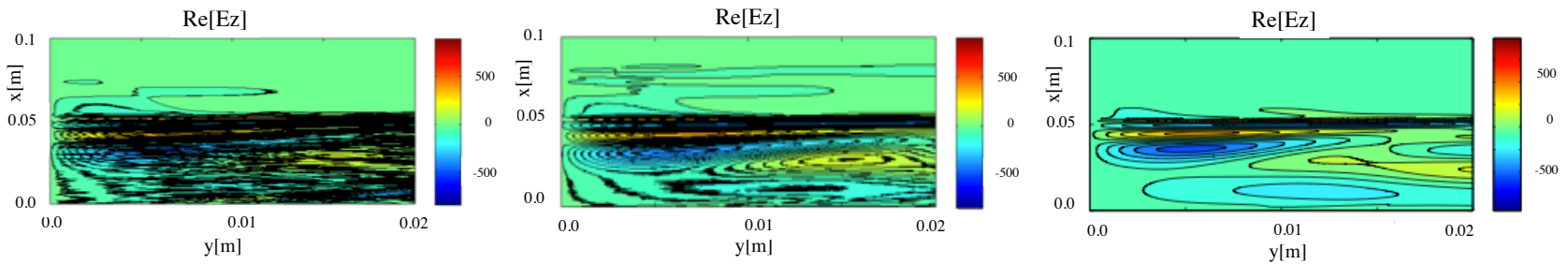

FIGURE 2. Improved resolution of the $\mathrm{E}_{z}$ component in the zone below the LH layer, i.e. where the slow wave has a propagative character.

set to be metallic plates, i.e. the tangential electric field components $\equiv 0$, at $x=0.0 \mathrm{~m}, y=0.0 \mathrm{~m}$ and $y=\mathrm{L}$. At the mouth of the antenna box $(x=\mathrm{H})$ it is assumed that the fields have a purely transverse electric character (TE). The following examples illustrate some of the phenomena that can occur in a real antenna box enclosed by metallic plates and in the presence of a finite plasma density. Examples for different electron densities $\left(\mathrm{n}_{e}\right)$ and density gradients are shown, including cases where the lower hybrid (LH) and/or ion-ion hybrid layer(s) lie inside the antenna box. In view of the fact that the density typically decays exponentially in the edge but connects to the core density inside the last close flux surface, in particular in H-mode, a fairly wide range of values of the densities $\left(\mathrm{n}_{e}=10^{15}-10^{20} \mathrm{~m}^{-3}\right)$ has been considered. The orientation between the static magnetic field and the electric field of the waves mixes slow and fast wave effects and influences the electric field pattern in the box. The equations are solved using a finite difference scheme, the number of grid points is typical of the order $1000 \times 1000$, which corresponds to a resolution of about $0.5 \mathrm{~mm}$. The adopted parameters are loosely inspired on JET parameters but have been adjusted to highlight specific issues. For illustration purposes some of the parameters are well outside the range for edge plasmas. 

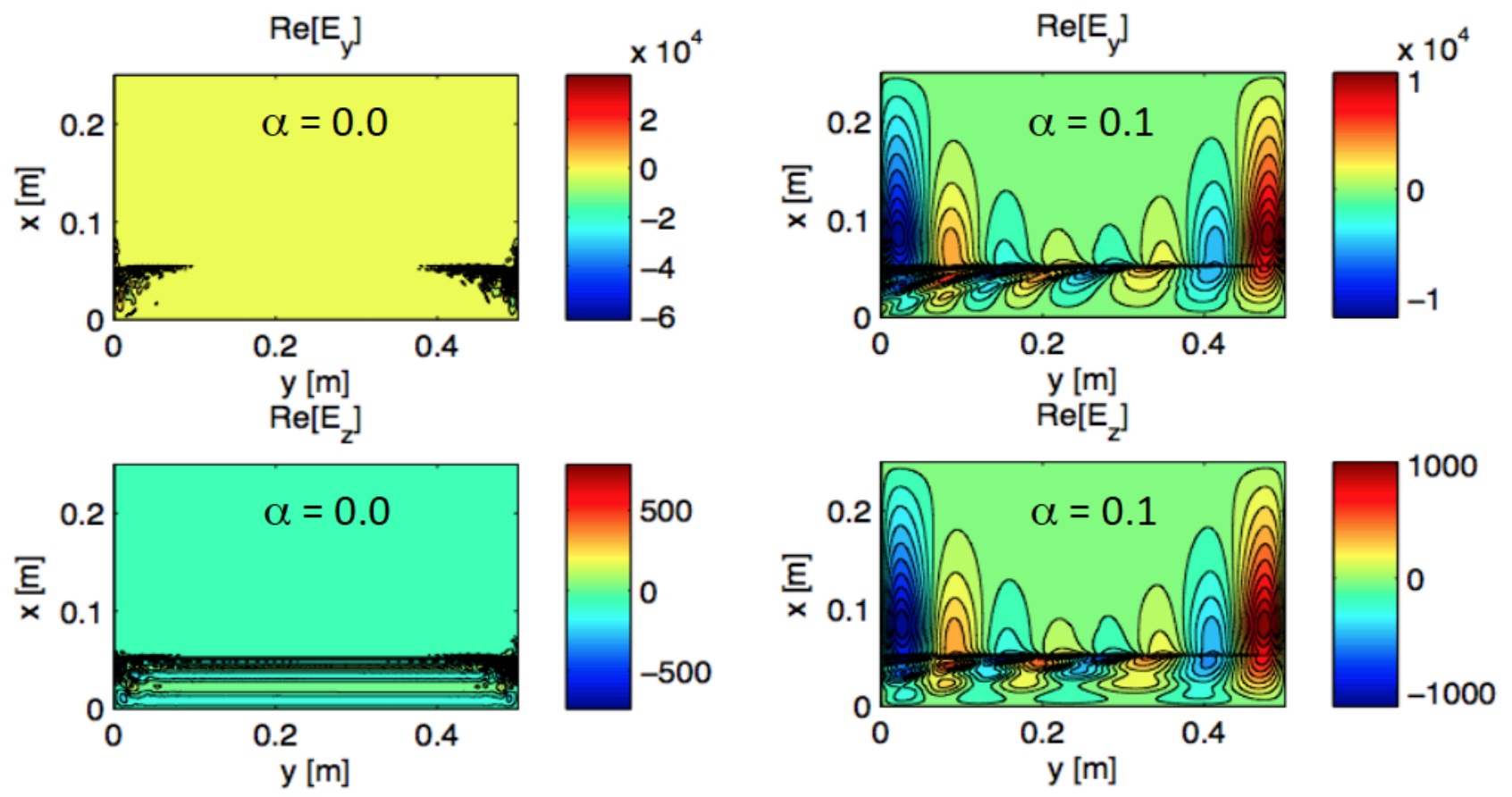

FIGURE 3. Real parts of $\mathrm{E}_{y}$ and $\mathrm{E}_{z}$ for $\alpha=0.0$ and $\alpha=0.1$
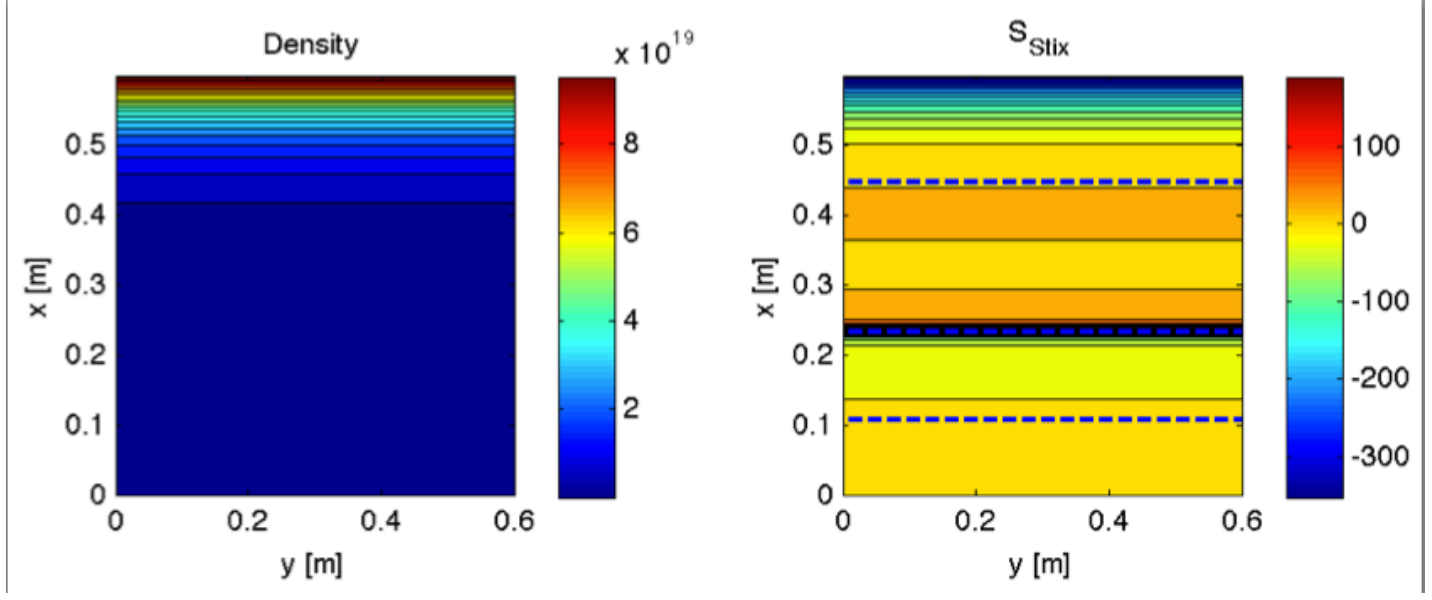

FIGURE 4. Density profile and Stix parameter $S$ for a simulation with variable magnetic field strength. The LH resonance $(\mathrm{x}=0.11 \mathrm{~m})$, the ion cyclotron layer for the $H$ minority $(\mathrm{x}=0.24 \mathrm{~m})$ and the ion-ion hybrid resonance $(\mathrm{x}=0.45 \mathrm{~m})$ are labelled by a dashed line.

\section{Influence of the plasma density}

The density and its gradient in the simulation domain determines the evanescent and propagative character of fast and slow waves. In the first example the influence of a strong variation in density over the height of the box is investigated, changing from $1 \times 10^{15}$ at $\mathrm{x}=0.0 \mathrm{~m}$ to $9 \times 10^{19} \mathrm{~m}^{-3}$ at $\mathrm{x}=\mathrm{H}$. The real parts of $\mathrm{E}_{y}, \mathrm{E}_{z}, \omega \mathrm{B}_{y}$ and $\omega \mathrm{B}_{z}$ are shown in figure 1. The background magnetic field was fixed to $3.25 \mathrm{~T}$, the antenna was placed at $x=0.20 \mathrm{~m}$. At $x \sim 0.08 \mathrm{~m}$ sits the lower hybrid resonance. As can be seen on the plot at the resonance layer the behaviour of the slow wave ( $\mathrm{E}_{z}$ component) changes from propagative to evanescence. The zone below the LH is surrounded by metallic plates and is a resonant cavity for the slow wave, which is numerically difficult to resolve. A sufficiently dense grid 

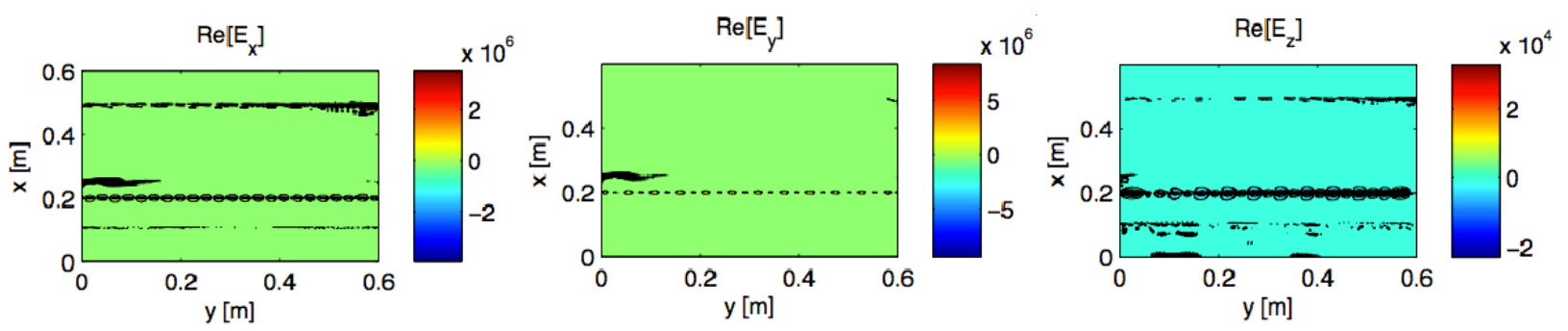

FIGURE 5. Real parts of $\mathrm{E}_{x}, \mathrm{E}_{y}$ and $\mathrm{E}_{z}$ for the run with variable magnetic field strength.

is required, as is illustrated in figure 2. The electric field of the slow wave component is shown for three different meshes in the zone below the LH layer. The number of grid points was increases from left to right, corresponding to a resolution in $(x, y)$ direction of $0.3 \mathrm{~mm} \times 0.6 \mathrm{~mm}, 0.2 \mathrm{~mm} \times 0.6 \mathrm{~mm}$, and $0.15 \mathrm{~mm} \times 0.5 \mathrm{~mm}$ respectively.

\section{Variation in orientation of the magnetic field}

If the angle between the magnetic field and the $\mathrm{z}$-direction is non-zero, both slow and fast wave components will be excited in the plasma even for an antenna current purely in $\mathrm{y}$ - or $\mathrm{z}$ - direction. A direct comparison of the resulting E-field components is shown in figure 3, the fields in the left column are for $\alpha=0$ (i.e. the magnetic field aligned to the $z$-direction) and the right column is for $\alpha=0.1$.

\section{Variation in magnetic field strength}

To illustrate the effect of an ion-ion hybrid resonance layer in the plasma, runs were performed with varying magnetic field strength, from $\mathrm{B}_{0}=3.15 \mathrm{~T}$ at $\mathrm{x}=0.0 \mathrm{~m}$ to $\mathrm{B}_{0}=3.70 \mathrm{~T}$ at $\mathrm{x}=0.60 \mathrm{~m}$. The antenna is located at $\mathrm{x}=0.20 \mathrm{~m}$. The density profile and S-number (as used in the Stix formulation [4]) are plotted in figure 4 . The three dashed lines indicate respectively the lower hybrid layer, characterized by $S=0$, located at $x=0.11 \mathrm{~m}$, the ion cyclotron layer for the $H$ minority at $\mathrm{x}=0.24 \mathrm{~m}$ and an ion-ion hybrid resonance $\left(k_{0}^{2} S=k_{\|}^{2}\right)$ at $\mathrm{x}=0.45 \mathrm{~m}$. The resulting electric field components are plotted in figure 5, with the LH, ion cyclotron and ion-ion hybrid layers at the correct $x$ locations.

\section{CONCLUSION}

It has been shown that both the slow and fast wave branches play a role in the RF wave propagation in cold plasmas with relatively low densities, as it is the case inside a sheath layer in front of a metallic surface and in the antenna box. Depending on the plasma density the fast and slow wave may be propagative or evanescent, and coupled or decoupled. Aside from the density variation which plays a dominant role, the waves respond sensitively to the plasma composition, and to the variation and orientation of the confining magnetic field. Numerical modelling quickly becomes demanding on computer power, since a fine grid spacing is required to capture the small wavelengths effects of strongly evanescent modes.

\section{REFERENCES}

1. D.A. D'Ippolito and J.R. Myra, Phys. Plasmas 13, 102508 (2006)

2. D. Van Eester, K. Crombé, V. Kyrytsya, Plasma Phys. Control. Fusion 55, 025002 (2013)

3. D. Van Eester, K. Crombé, V. Kyrytsya, Plasma Phys. Control. Fusion 55, 055001 (2013)

4. T.H. Stix, Waves Plasmas (New York: Springer) (1992) 